DIMENSIONAL

\section{OPTIMIZATION OF THE ROBOTIC ARM TO REDUCE ENERGY CONSUMPTION}

VLADIMIR MOSTYN ${ }^{1}$, DANIEL HUCZALA ${ }^{1}$, WOJCIECH MOCZULSKI², ANNA TIMOFIEJCZUK ${ }^{2}$

${ }^{1}$ VSB - Technical University of Ostrava, Faculty of Mechanical Engineering, Dept. of Robotics, Ostrava, Czech Republic

${ }^{2}$ Silesian University of Technology, Faculty of Mechanical Engineering, Institute of Fundamentals of Machinery Design, Gliwice, Poland

DOI: 10.17973/MMSJ.2020_03_2020001 vladimir.mostyn@vsb.cz

This study examines selected components of the optimization function, used to evaluate the optimal kinematic structure of a robot for a given task. Automated generation of the kinematic structure is based on scalable drive modules of the joints and modules of the carrying arms with a check on the permissible torque of the drive and bending moment of the carrier element. An optimization algorithm is used to generate variations of kinematic structures, the base requirement of the fitness function is the ability to traverse a given trajectory with a defined orientation of the tool. The suitability of a given kinematic structure is evaluated further by a set of evaluation functions such as a check for spatial collisions, energy consumption, minimization of total weight, minimization of degrees of freedom for a given task and several other criteria. Two of these criteria - evaluation of the total weight of a robotic arm with drives in joints and evaluation of power consumption for a defined handling task are examined here.

KEYWORDS

Generation of the robot kinematic structure, conceptual design, optimization, fitness function, energy consumption.

\section{INTRODUCTION}

This paper focuses on automatically generated structures of manipulation arms that were created from databases of suitable actuators, links and grippers. This goal was created primarily as a supporting software tool for manufacturing system designers who use sets of industrial building blocks and it can also be applied to automatically assembled modular handling systems to achieve a desired handling task. Definition of some spatial restrictions on the desired handling system is an optional choice. Here we deals with selected components of the evaluation function used to generate an appropriate kinematic structure of the robot at a given position of the robot base and with a specified tool trajectory including its orientation. Generation of a kinematic structure is based on scalable joint drive modules and scalable carrying links with a check of the permissible torque of the drive and bending moment of the carrier element. In the first phase of the project, intelligent functional modules of Schunk rotary joints were used to generate the kinematic structure. Modules are optionally interconnected either by catalogue link modules or by custom modules with spatial variability in length or in orientation with checking of adherence to the permitted torque of the drive or carrying module. Within the project, however, custom scalable joint modules for rotational and translational motion are being developed. The principle means of generating a kinematic structure is a genetic algorithm that generates individual generations of kinematic structures. The base criterion is the ability to pass a given trajectory with defined tool orientation. Another major criterion is a check for spatial collisions of individual rotating modules and their links and the check of torques of individual drive modules. Additional criteria for assessing a given kinematic structure are a set of weighted complementary evaluating functions, such as energy consumption, weight minimization, minimization of degrees of freedom for a given task and several other criteria. Two of these criteria - evaluation of the total weight of a robotic arm with joints and their drive units and evaluation of power consumption for a defined handling task are examined in this study. The purpose of the paper is not to examine the optimization algorithm, but only to examine selected components of the so called fitness function (also called cost function), when evaluating total weight of a robotic arm with joints and their drive units and evaluating power consumption for a defined handling task as a function of the links lengths ratios.

In the available literature, the area of optimization of manipulation tasks is concentrated for practical reasons primarily on minimizing energy consumption for a given handling task. There are three basic approaches - optimization at the level of local trajectory planning, i.e. optimization of velocity and acceleration along trajectory, jerk minimization and its continuity in individual trajectory segments. In [Björkenstam 2013] a path planning algorithm is introduced that calculates an initial collision free path using a convex optimal control, then a nonlinear optimal control is used to iteratively improve the trajectory. In [Porawagama 2014] the authors introduce a new trajectory planning method for generating bounded and continuous jerk trajectories in joint space using polynomial segmented 5-3-5 splines. In [Chen 2011] the authors propose computational techniques to find the maximal acceleration and minimal jerk along the trajectory and in [Zhang 2017] a new robot trajectory planning method is introduced based on a genetic chaos optimization algorithm. An algorithm for jerk bounded Synchronized Trigonometric S-curve Trajectory (STST) and the 'forbidden-sphere' technique to avoid obstacles have also been proposed in [Perumaal 2013] and in [Komak 2018] authors propose smoothed collision-free trajectory around the obstacles.

A second group of work deals with optimization of the relative position and orientation of the desired trajectory toward the robot as presented in [Luo 2018]. A third approach is to optimize the power consumption of more robots cooperating within the robotic cell or whole line, optimizing the trajectory, velocity profile, cycle length, braking time and other parameters. In [Vergnano 2012] authors presented an energy-optimal schedule, derived by solving a mixed-integer nonlinear programming problem. It has been shown that by using automatic path planning and line balancing instead of standard offline programming the cycle time in welding lines can be improved by as much as $25 \%$ [Björkenstam 2013]. A very advanced and 
practically validated method is presented in [Bukata 2017], where the authors' hybrid heuristic algorithm was accelerated by using multicore processors and the Gurobi Optimizer.

The emerging use of automated robotic systems and the need for automatic reconfiguration of handling systems when changing the manipulation task give these optimizations a new relevance with a strong emphasis on the weight of the resulting system and the associated energy consumption to perform a given handling task.

Optimizing energy consumption is also becoming increasingly important for mobile robotic devices that have limited energy supplies. Numerous works have addressed the optimization of the kinematic structure of robotic mechanisms in terms of joints payload optimization and force transmission. A foot optimization of the hexapod's leg utilizing an objective function that considers both dexterity and payload is introduced in [Xin 2015]. Energy consumption and load on joints are also significantly influenced by the current robot configuration - here configuration means one of the possible solutions of the inverse kinematics as is shown in [Gouasmi 2012]. These optimization tasks, however, use the fixed structure of the mechanism with given mass parameters, while we are concerned with creating a "tailor-made" mechanism for a given manipulation task by selecting carrying elements of optimal length or length ratio and optimal driving elements from a database.

Finding the optimal ratio of lengths of individual links of the manipulator is based on a dynamic model of the manipulating arm with variable lengths of individual links, which is processed using the Matlab simulation system. The correctness of the dynamic model calculation was verified for selected arm configurations in the environment of the CAD software Creo Mechanism. This approach is often used because Matlab allows for greater programming variability than multibody dynamics of CAD systems. The simulation model was designed so that not only the length of individual links is varied, but for each modified link length a new dimensioning of the supporting link and the drive is performed and the effect of the individual link lengths on the total weight of the manipulator and also on the energy consumption are evaluated for the reference handling task. Comparisons between kinematic structures with random lengths of robot supporting links with a given total length are meaningless. A suitable approach seems to be the generation of varying relative lengths of individual links of the reference model and finding the optimal ratio of the lengths of links to minimize both total weight and energy consumption. The aim is to target another component of the evaluation function (fit function, cost function) for the algorithm of genetic generation of robot kinematic structures and to analyses its total mass. It is obvious that the weight of this criterion must be reduced compared to the main goal - to reach all points of the desired trajectory with the required tool orientation. In an environment with spatial limitation of arms by obstructions, mass optimization of link length is even more challenging, because the length of the links is primarily determined by the spatial possibilities of the work space. The question is whether there is any optimum of suitable lengths of links, here lengths ratio, for minimum weight and energy consumption and whether this minimum is significant enough and worth applying during automatic mechanism design.
In Part 1, we discuss the appropriate reference model of the manipulating arm to examine energy consumption and choice of handling task. In part 2, we analyses the selected type of drive module and make a synthesis of the so-called continuous mathematical model of the drive module. In Part 3, we present a synthesis of the individual links and their dimensioning based on adherence to the permissible bending stress for the given cross-section and material of the link. In Part 4, we present a simplified dynamics model of a given manipulator structure and analyses weight parameters for varying ratios of individual link lengths and evaluate energy consumption for the reference handling task.

\section{REFERENCE MODEL OF THE MANIPULATION ARM}

Based on the analysis of physical phenomena related to energy losses in individual robot subsystems, it can be stated that only dissipative forces cause loss in the mechanical subsystem of the manipulator, specifically frictional losses in the mechanism joints, gears and motors, and losses caused by aerodynamic drag during movement. To determine these losses, it is necessary to build a dynamic model of the mechanism, determine the load forces and torques in individual joints and determine the corresponding frictional forces and torques. Besides the losses due to dissipative forces, there is no energy loss in the mechanism when moving within the working cycle, only changes in potential energy and kinetic energy, and their mutual transformation, occur during motion. When a trajectory is a closed path the sum of these energies is equal at the start and end point of the movement. For example, energy coming from the drive system accumulates to increase the potential energy, which in turn returns to the drive system when returning to its initial position. Similarly, kinetic energy accumulates in the movement of the links, gears, and engine rotors during acceleration, and returns to the drive system during braking. In physical terms, we can talk about power sign and the associated drive system's ability to recover negative power back to the power supply system. If the drive system is unable to recuperate power to the grid, or at least to a common DC link, braking losses are mostly dissipated by braking resistors and form a significant part of the energy consumption of the entire system.



Figure 1 Reference model of the manipulation arm 
Another place where losses occur in any direction of the energy flow, even at the steady position of the mechanism (at zero speed with torque holding) are the resistances $R_{a}$ of the electric motor windings. A motor torque (positive when accelerating, negative when braking) is proportional to the current $I$ of the motor, the proportional constant being the torque constant. Thermal power losses $P_{t h}$ of the winding are independent of current direction, and are equal to $P_{t h}=R_{a} \cdot I^{2}$ and during the process they change into heat energy produced by the motor. These winding heat losses are about 3 to 5 percent of the motor rated power and thus represent a relatively small portion of the energy consumption to perform a handling task. In any case, the highest energy consumption and hence energy losses are observed when the highest torques of the drives are needed during acceleration of the links. This happens unambiguously with the highest extension of the arm and when accelerating upwards against gravity. Therefore, the simplified mechanism of the manipulation arm with 3 degrees of freedom (DOF), shown in Figure 1, was chosen as the reference calculation model. The position at which all the links are horizontal and move upward against gravity was chosen as the starting position. In order not to consider the amount of energy recovery, we selected the trajectory where individual links accelerate with constant angular acceleration as the reference manipulation task. Figure 1 shows the positions of the links when the handling task is finished. The model consists of an angular kinematic structure with 3 DOFs. This structure with 3 angular joints is widely used not only in service robotics, but it is also very common in industrial robotics. This concept may be easily supplemented by other DOFs, e.g. vertical axis in the base of a robot or wrist axes. Alternatively, the analysis of the effect of the link's length ratio on the total weight of the mechanism and on the energy consumption can be based on any starting position of the mechanism. In the case of the "folded" position of the mechanism however, where some links may be near the vertical position, the effect of the lengths of the links will be significantly smaller. The same is true for a different orientation of the joint axes, for example, for the kinematic structure of the "scara" type, the influence of the weight of the drives will be considerably smaller.

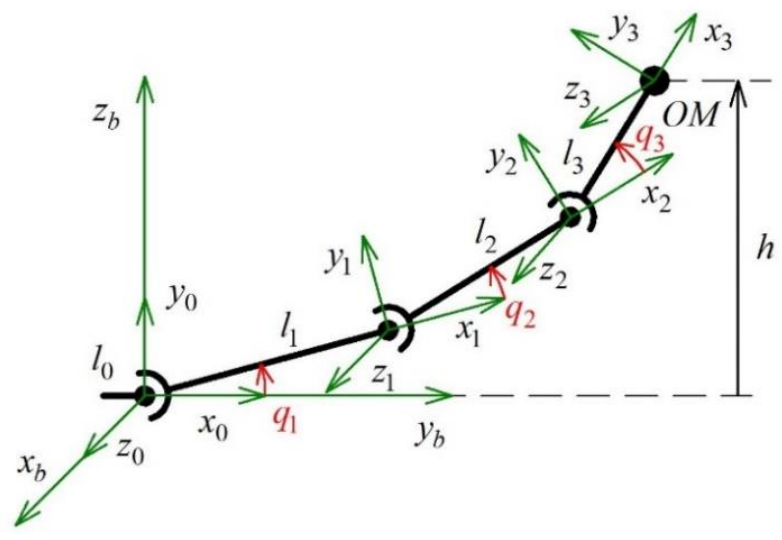

Figure 2 Kinematic structure with local coordinate systems

Since we want to investigate the influence of the links length ratio on energy consumption, and the end point trajectory changes slightly as the length of individual links changes, the reference manipulation task is defined as raising the object of manipulation OM with a mass $m_{O M}$ to a height $h$ during time $t_{\text {max }}$ with constant angular acceleration of the all links $\varepsilon$. Initial parameters of the task are the required mass of the object $m_{O M}$ and the required range of the handling arm, which defines the total length $l_{c}$ of the handling arm as the sum of the individual link's lengths. This handling task is investigated for different link length ratios and energy consumption is evaluated as a change in the sum of kinetic and potential energy of the entire system. In the starting position, all the links are in a horizontal position in which the highest bending moments are caused by gravitational forces and thus the highest tensile stress is exerted at the connection point of the link and the joint and at the same time the highest load torques are applied to the drives. The kinematic structure of the reference model is shown in the Figure 2. Local coordinate systems were defined using DenavitHartenberg notation. As mentioned above, the change in length of a given link is not only reflected by the change in the torque that the drive has to develop, but also the cross section of the link is adapted to the changed tilting moment according to the maximal permissible stress $\sigma$ in the carrier element in the calculation cycle. The drive size is adjusted to the needed torque taking into account the reduced moment of inertia of the motor and gearbox.

The arm links in the reference model are tubes of radius $R$ with wall thickness $s$, of material aluminum alloy Al 6061. The crosssection dimensions may vary in two ways: by changing radius $R$ while keeping thickness $s$ constant or changing the $s$ while keeping $R$ constant. The first method is preferable, with the possibility of entering a suitable wall thickness according to market availability. The second method of increasing the wall thickness of the tube at the same diameter is less advantageous because it leads to higher link weights at the same load capacity. Increasing the diameter results in a higher weight of the support element, which in turn causes an increase in the bending moment. Thus, the process is iterative and a new outer tube diameter is calculated for each link length so that the maximum tensile stress $\sigma_{\max }$ at the point of attachment of the link to the joint is constant and less than the allowable stress with some safety factor.

The task is further complicated by the mass parameters of the drive located in the joint - its mass and reduced moment of inertia. The mass of the drive loads the link by bending moment and increases the required drive torque in the previous joint, while the reduced moment of inertia of the motor-gearbox is added to the moment of inertia of the following link and increases the torque in the joint needed to accelerate the link. The weight of the motor is often not such a problem as the moment of inertia of the rotor. Reducing even the low moment of inertia of the rotor to the gearbox output is proportional to the square of the transmission gear ratio, and thus a relatively small moment of inertia at high gear ratio and at high accelerations causes a high dynamic torque that the drive must overcome.

In this way, the designer comes to a situation where they propose pre-drives for the lengths of the links found in the kinematic structure and for the given acceleration and endeffector load, which increases the weight of the links by the drives' weights and increases the required torque in the joint by the motor and gearbox reduced moment of inertia. This will require larger drives and/or larger gears which, by their reduced 
moment of inertia result in a further increase in the required torque in the joint and, again, in larger drives and more massive links with higher carrying capacity.

Often, this iterative process does not have a solution for a given length, required load capacity, and especially for the desired acceleration, and after several laborious iterations, the designer is forced to compromise the input requirements for accelerating the links.

\section{CONTINUOUS DRIVE MODEL}

In order to optimize the calculation of the arm system with actuators in the joints, it was necessary to create a physical interpretation of the motor and gearbox assembly and create a "continuous" mathematical model of the drive. The continuous mathematical model expresses the relationship between mass and reduced moment of inertia of the drive on one site and the maximal allowed output torque of the drive on the other site. This functional dependency is based on the analysis of real motors of the manufacturer TG Drives (motors type TGT) connected to gearboxes of Harmonic Drive manufacturer (HDUR-20-IH to HDUR-100-IH). These units were used during design, assembly and production of the heavy manipulation arm of the mobile robot Hardy [Pozary.cz 2011] that is supposed to help fire fighters during their missions.

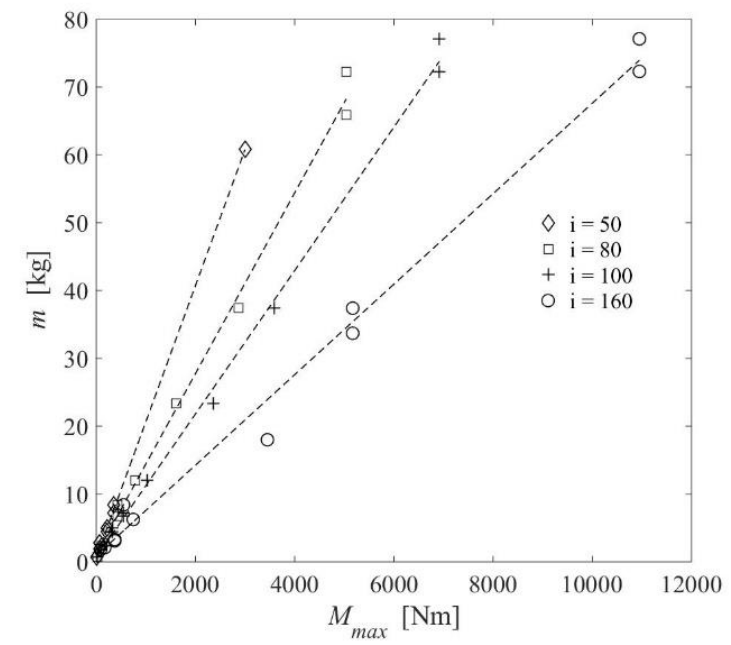

Figure 3 Total mass of drive unit related to peak torque of selected combinations

The motors and gearboxes were coupled into suitable combinations considering a range of nominal and peak torques. The total mass of a drive unit was calculated as the sum of the motor mass and gearbox mass and reduced moments of inertia were calculated for these drives using the gear ratio. Figures 3 and 4 show values of total masses and reduced moments of inertia of the drives related to peak torque for all the drive unit combinations. The results are particularly significant for harmonic gearheads. Using regression analysis, straight lines can be used to approximate drive weight curves based on maximum output torque for individual gear ratios. Individual points on the corresponding graph indicate catalogue values of the sum of motor and gearbox weights. For some torque sizes, there are two points in the graph because two different motor and gearbox combinations correspond to the respective maximum output torque.

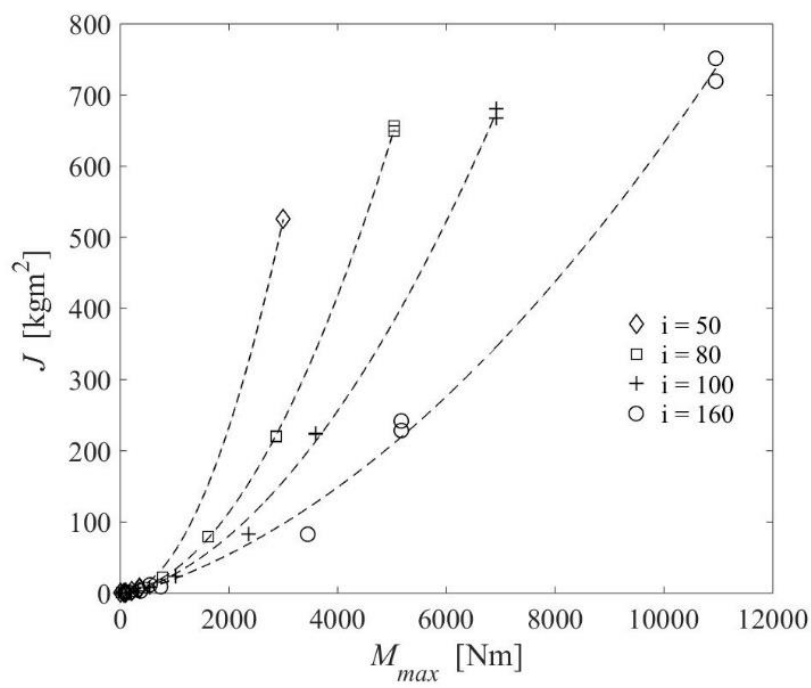

Figure 4 Reduced moment of inertia related to peak torque on gearhead's output of selected combinations

Regression line of the drive unit with the ratio $\mathrm{i}=50$ is extrapolated for comparison. The regression lines are represented by equations (1) to (4).

$$
\begin{aligned}
& \mathrm{m}_{50}=0.0201 M_{\text {max }}+0.6009 \\
& \mathrm{~m}_{80}=0.0128 M_{\text {max }}+1.4611 \\
& \mathrm{~m}_{100}=0.0106 M_{\text {max }}+0.6697 \\
& \mathrm{~m}_{160}=0.0067 M_{\max }+0.9225
\end{aligned}
$$

A regression for values of the reduced moment of inertia related to peak torque on the gearhead's output was done in a similar way using a second degree polynomial function. The distribution is represented by equations (5) to (8). Again, the distribution of moment of inertia related to gear ratio $i=50$ is extrapolated to see the results more clearly.

$$
\begin{aligned}
& \mathrm{J}_{50}=6.550 E-05 . M_{\text {max }}^{2}-3.943 E-03 . M_{\text {max }}+4.860 E-01 \\
& \mathrm{~J}_{80}=2.393 E-05 . M_{\text {max }}^{2}+8.779 E-03 . M_{\text {max }}+4.073 E-02 \\
& \mathrm{~J}_{100}=1.135 E-05 M_{\text {max }}^{2}+1.979 E-02 M_{\text {max }}-4.497 \\
& \mathrm{~J}_{160}=4.198 E-06 M_{\text {max }}^{2}+2.190 E-02 M_{\text {max }}-5.961
\end{aligned}
$$

It can be seen from the Figure 3 and Figure 4 that the choice of a higher ratio is a more advantageous solution for given torque at the gearbox output, as it results in lower overall drive weight and a lower reduced moment of inertia. However, this is only true for harmonic gearboxes. With the use of conventional gearboxes with higher ratios, the number of gears increases and thus considerably increases their weight and moment of inertia. The presented mathematical model of a drive has a certain disadvantage in its "continuity". The continuity is contrary to discrete sizes of real motors and gears that are always manufactured in a series. On the other hand, this approach brings an easier convergence of the optimization model when 
searching a manipulator's parameters. For the actual solution, the closest available drive in the manufacturer's range must be used and all related parameters rechecked. In the simulation model, gears with a ratio of $i=160$ were used and for calculation purposes the absolute term in relation (8) was zeroed.

\section{COMPUTATIONAL MODEL OF MECHANISM DYNAMICS}

The rigid links and drives of the manipulation arm are dimensioned for a given handling task of raising the manipulation object OM to a height $h$ in time $t_{\max }$ with a constant angular acceleration $\varepsilon$ of individual joints as is shown in Figure 2. The load of the of the arm links and drives is calculated as the sum of static and dynamic moments. Calculation of the parameters of bearing and driving elements of the manipulator is performed repeatedly for different lengths of individual manipulator links. The links lengths are $l_{1}, l_{2}, l_{3}$, total length of the manipulator is $l_{c}=l_{1}+l_{2}+l_{3}$. The length ratio between the links is described by the coefficient $k$, so the lengths of individual links can be expressed as:

$$
l_{1}=\frac{l_{c}}{k^{2}+k+1} \quad l_{2}=l_{1} \cdot k \quad l_{3}=l_{2} \cdot k
$$

The coefficient $k$ is changed in the program cycle within a defined range - e.g. 0.5 to 2.0 with a defined step. For each link's length, the angular acceleration $\varepsilon$ of the links is first calculated to achieve the desired height $h$ in time $t_{\max }$ with constant link angular acceleration $\varepsilon$. The position of the first link for uniformly accelerated motion can be calculated from the equation

$$
q_{1}=\alpha=\frac{1}{2} \varepsilon \cdot t_{\max }^{2}
$$

The angular acceleration of individual links can be calculated on the basis of goniometric relations according to Figure 5, but the calculation leads to a numerical solution of the transcendental equation according to equations (11) and (12).

$$
h=l_{1} \sin (\alpha)+l_{2} \sin (2 \alpha)+l_{3} \sin (3 \alpha)
$$

$$
h=l_{1} \sin \left(\frac{1}{2} \varepsilon \cdot t_{\max }^{2}\right)+l_{2} \sin \left(\varepsilon \cdot t_{\max }^{2}\right)+l_{3} \sin \left(\frac{3}{2} \varepsilon \cdot t_{\max }^{2}\right)
$$



Figure 5 Computing of the links angles
The use of inverse kinematics to calculate link angles is not appropriate here, since the mechanism is in its singular position, and in addition, endpoint trajectories vary with variable link lengths. Therefore, an elegant solution of the direct kinematics is to use a simple iterative calculation of the product of exponentials. Initial angular acceleration $\varepsilon=0.0\left[\mathrm{rad} / \mathrm{s}^{2}\right]$ is increasing in a cycle until the OM achieves desired height $h$ in a time $t_{\max }$. The links are moving with continuous angular acceleration and during this process the system is continuously calculating the $z$-coordinate of the OM. The $z$-coordinate is a component of the homogenous transformation matrix $\mathbf{T}_{b 3}$ between the reference coordinate system $x_{b}, y_{b}, z_{b}$ and the last coordinate system $x_{3}, y_{3}, z_{3}$ in the center of OM.

The algorithm is as follows:

1. Initialization - common angular acceleration of the joints is set to zero, height $z$ is set to zero

$$
\varepsilon=0, z=0, \text { step }=0.001\left[\mathrm{rad} / \mathrm{s}^{2}\right]
$$

In accordance with Figure 2, the "zero configuration" transformation matrix from the base coordinate system $x_{b}, y_{b}, z_{b}$ to the coordinate system of the OM $x_{3}, y_{3}, z_{3}$ is

$\mathbf{M}=\left[\begin{array}{llll}1 & 0 & 0 & 0 \\ 0 & 1 & 0 & l_{c} \\ 0 & 0 & 1 & 0 \\ 0 & 0 & 0 & 1\end{array}\right]$

The screw vector and screw matrix are defined as

screw vector $\mathbf{S}_{i}=\left[\begin{array}{c}\boldsymbol{\omega}_{i} \\ \mathbf{v}_{i}\end{array}\right]$

screw matrix $\hat{\mathbf{S}}_{i}=\left[\begin{array}{cc}\hat{\boldsymbol{\omega}}_{i} & \mathbf{v}_{i} \\ \mathbf{0}^{T} & 0\end{array}\right]$

where $\hat{\boldsymbol{\omega}}_{i}$ is the skew vector of $\boldsymbol{\omega}_{i}$. Components of the screw vectors expressed in the base frame are shown in Table 1:

\begin{tabular}{l|ll|ll|l}
$\mathbf{i}$ & \multicolumn{2}{|l}{$\boldsymbol{\omega}_{i}$} & \multicolumn{2}{l}{$\mathbf{p}_{i}$} & \multicolumn{2}{l}{$\mathbf{v}_{i}$} \\
\hline $\mathbf{1}$ & {$\left[\begin{array}{lll}1 & 0 & 0\end{array}\right]^{T}$} & {$\left[\begin{array}{lll}0 & 0 & 0\end{array}\right]^{T}$} & {$\left[\begin{array}{lll}0 & 0 & 0\end{array}\right]^{T}$} \\
\hline $\mathbf{2}$ & {$\left[\begin{array}{lll}1 & 0 & 0\end{array}\right]^{T}$} & {$\left[\begin{array}{lll}0 & l_{1} & 0\end{array}\right]^{T}$} & {$\left[\begin{array}{lll}0 & 0 & -l_{1}\end{array}\right]^{T}$} \\
\hline $\mathbf{3}$ & {$\left[\begin{array}{lll}1 & 0 & 0\end{array}\right]^{T}$} & {$\left[\begin{array}{llll}0 & l_{1}+l_{2} & 0\end{array}\right]^{T}$} & {$\left[\begin{array}{lll}0 & 0 & -\left(l_{1}+l_{2}\right)\end{array}\right]^{T}$}
\end{tabular}

Table 1. Components of the screw vectors

where translational velocity vector is computed as

$$
\mathbf{v}_{i}=-\boldsymbol{\omega}_{i} \times \mathbf{p}_{i}
$$

2. While $z \leq h$ increase acceleration $\varepsilon$ by step , $\varepsilon=\varepsilon+$ step 
when the condition isn't true go to step 6 - end of iteration.

3. Actual joints positions are computed as

$$
q_{1}=\frac{1}{2} \varepsilon \cdot t_{\max }^{2} q_{2}=\frac{1}{2} \varepsilon \cdot t_{\max }^{2} q_{3}=\frac{1}{2} \varepsilon \cdot t_{\max }^{2}
$$

4. The transformation matrix from the base coordinate system to the coordinate system of the OM can be computed as a product of exponentials

$$
\mathbf{T}_{b 3}\left(q_{1}, q_{2}, q_{3}\right)=e^{\hat{\mathbf{S}}_{1} \cdot q_{1}} \cdot e^{\hat{\mathbf{S}}_{2} \cdot q_{2}} \cdot e^{\hat{\mathbf{S}}_{3} \cdot q_{3}} \cdot \mathbf{M}
$$

5. The $z$-coordinate of the OM can be computed as the element of the transformation matrix $\mathbf{T}_{b 3}$

$$
z=\mathbf{T}_{b 3[3,4]}
$$

Return to the step 2

6. End of iteration, $\varepsilon$ contains the desired angular acceleration of the joints.

The load calculation of the individual links begins with the last link. Based on the known mass $m_{O M}$ of the OM and given link length $l_{3}$ in the iteration cycle, the outer diameter $R_{3}$ of the link tube is consecutively increased from a minimum value until the bending stress at the connection point is less than the allowable stress of the given material, or the desired link stiffness can be used here. This yields a cross section of the supporting tube of the link and for the known length $l_{3}$ of the link, the mass $m_{3}$ and the position $\mathbf{p}_{c 33}$ of the center of gravity of the link 3 (including OM) are expressed in the third local coordinate system of the last link

$$
\mathbf{p}_{c 33}=\left[\begin{array}{c}
\frac{-l_{3}}{2} \cdot m_{3} /\left(m_{O M}+m_{3}\right) \\
0 \\
0
\end{array}\right]
$$

with the components of $x_{c 33}, y_{c 33}$ and $z_{c 33}$. The next step is calculation of the inertia matrix of the third link (including $O M$ )

$$
\mathbf{J}_{3 O M c}=\left[\begin{array}{ccc}
J_{3 O M x} & 0 & 0 \\
0 & J_{3 O M y} & 0 \\
0 & 0 & J_{3 O M z}
\end{array}\right]
$$

where the following equations determine the moments of inertia related to the center of gravity of the third link including OM:

$$
\begin{aligned}
& J_{3 O M x}=J_{3 c x}+J_{O M} \\
& J_{3 O M y}=J_{3 c y}+m_{3}\left(\frac{l_{3}}{2}+x_{c 33}\right)^{2}+J_{O M}+m_{O M} \cdot x_{c 33}^{2}
\end{aligned}
$$

$$
J_{3 O M z}=J_{3 c z}+m_{3}\left(\frac{l_{3}}{2}+x_{c 33}\right)^{2}+J_{O M}+m_{O M} \cdot x_{c 33}^{2}
$$

The moments of inertia of the carrying tube and OM (that has a spherical shape) are expressed in the following equation

$$
\begin{aligned}
& J_{3 c x}=m_{3}\left(R_{3}^{2}+r_{3}^{2}\right) / 2 \\
& J_{3 c y}=J_{3 c z}=m_{3}\left(3 R_{3}^{2}+3 r_{3}^{2}+l_{3}^{2}\right) / 12 \\
& J_{O M}=\frac{2}{5}\left(m_{O M} r_{O M}^{2}\right)
\end{aligned}
$$

Using Newton-Euler recurrent equations the vector of the translational acceleration $\mathbf{a}_{c 3}$ at the center of gravity and vector of the angular acceleration $\boldsymbol{\varepsilon}_{3}$ are calculated. Based on these values an action torque $\mathbf{n}_{3}$ and an action force $\mathbf{f}_{3}$, that load the joint between the second and third link, can be determined as follows in equations (28) and (29).

$$
\begin{aligned}
& \mathbf{f}_{3}=m_{3}\left(\mathbf{G}-\mathbf{a}_{c 3}\right)+\mathbf{f}_{4} \\
& \mathbf{n}_{3}=\mathbf{n}_{4}+\mathbf{N}_{3}+\left(\mathbf{p}_{23}+\mathbf{p}_{c 3}\right) \times \mathbf{f}_{3}-\mathbf{p}_{c 3} \times \mathbf{f}_{4}
\end{aligned}
$$

where

$$
\mathbf{G}=\left[\begin{array}{c}
0 \\
0 \\
-g
\end{array}\right] \quad g=9.80665\left[\mathrm{~ms}^{-2}\right]
$$

is a vector of gravitational acceleration

$$
\mathbf{N}_{3}=-\left[\mathbf{J}_{3 O M c} \cdot \varepsilon_{3}+\omega_{3} \times\left(\mathbf{J}_{3 O M c} \cdot \omega_{3}\right)\right]
$$

is inertial torque caused by tangential and centrifugal accelerations, $\mathbf{p}_{23}$ is a position vector from the origin of the 2 nd coordinate system to the origin of the 3rd local coordinate system expressed in the reference (basic) coordinate system and $\mathbf{p}_{c 3}$ is a position vector of the center of gravity of the 3rd link $\mathbf{p}_{c 33}$ also expressed in the reference coordinate system. When evaluating the balance of forces and moments of the last link, there are zero values of the torque $\mathbf{n}_{4}$ and force $\mathbf{f}_{4}$ acting to the object of manipulation OM. Mass properties of the OM are already included in the mass properties of the third link. The stress value $\sigma_{3}$ is calculated in the connection of a link and a joint

$$
\sigma_{3}=\frac{\left|\mathbf{n}_{3 z}\right|}{W_{o 3}}
$$

where $W_{o 3}$ stands for the cross-section module of the tube

$$
W_{o 3}=\frac{\pi\left(R_{3}^{4}+r_{3}^{4}\right)}{4 R_{3}}
$$


A projection of the torque $\mathbf{n}_{3}$ into the rotation axis $z_{2}$ is a generalized force $\tau_{3}$ that must be produced by the drive. The results of this iteration step are mass properties of the third link including $\mathrm{OM}$ and the torque required to move the third link with given acceleration and that loads the drive of the third link. The iterative calculation of the drive parameters follows in the next step. First, the starting parameters of the drive are found based on the relationships (4) and (8) for the load torque calculated in the previous step - the starting drive weight $m_{p 3}$ and starting reduced moment of inertia $J_{3 m p}$. While the mass $m_{p 3}$ of the drive is held by the previous second link, the reduced moment of inertia $J_{3 m p}$ increases the moment of inertia of the third link and must be added to the moment of inertia of the third link around the rotation axis $z_{2}$ according to equation (34).

$$
J_{3 O M z}=J_{3 c z}+m_{3}\left(\frac{l_{3}}{2}+x_{c 33}\right)^{2}+J_{O M}+m_{O M} \cdot x_{c 33}^{2}+J_{3 m p}
$$

As the total moment of inertia of the third link increases by adding the reduced moment of inertia of the drive, the torque needed to be exerted by the drive for the given acceleration must be recalculated. This leads to its enlargement and to the higher weight and the higher moment of inertia of the third drive that is added again to the inertia of the third link. This calculation is iterated as long as the required drive torque increases. After the iterative calculation for the third drive, the complete mass properties of the third link are known - its mass and moment of inertia including OM and the mass properties of the third drive its mass, which loads the second link and the reduced moment of inertia, which must be added to the moment of inertia of the third link.

Calculation of the loads of the second and the first link are analogous, instead of the mass $m_{O M}$ in the equations (18) and (20) to (22) we use the masses of motors $m_{p 3}$ and subsequently $m_{p 2}$, the link is loaded on its end by the action torque $\mathbf{n}_{3}$ and by the action force $\mathbf{f}_{3}$ caused by the third link, subsequently by the torque $\mathbf{n}_{2}$ and by the force $\mathbf{f}_{2}$ caused by the second link.

An iterative approach is used again for the calculation of an optimal cross-section of the carrier-link and for determining a size of a motor, whose reduced moment of inertia is always added to the moment of inertia of the appropriate link. The mechanism is examined in the initial position when the forces and torques achieve their peaks. Masses of the joints are determined using the coefficient multiplying the mass of the motors by a value of $k_{m p}=1.5$. Finally, the value of the mechanical work that has to be spent to lift OM to a given height over a set time is calculated. The work is calculated as the sum of kinetic and potential energy of all manipulator bodies, including drives. The work of dissipative forces is not included here. Correctness of calculation of load forces and joint torques and simulation of the mechanism's dynamics were verified in CAD system Creo 5.

\section{SIMULATION RESULTS}

The simulation model was performed in Matlab as a script. The presented results correspond with input values of mass of manipulated object $m_{O M}=50 \mathrm{~kg}$, total length of the manipulator $l_{c}=2 \mathrm{~m}$ and height $h=1 \mathrm{~m}$ representing the final position that must be achieved by the manipulator with a constant angular acceleration in time $t_{\max }$. In order to obtain load results for different accelerations, the cycle time $t_{\max }$ is consecutively reduced in the program cycle, this leads to an increase in the angular acceleration of the links up to the limit, where a drive solution is no longer found for the given acceleration. In the inner loop, the links length ratio is varied within a specified range - in this case 0.5 to 2.0 .

The main observed objective - finding such a ratio of links lengths which minimizes energy consumption or the total weight of the manipulator - was not achieved. As an example, Figure 6 shows the overall weight of the manipulator and energy consumption of the given manipulation task as a function of the links length ratio. The angular acceleration of the links in this case is $\varepsilon=1.03[\mathrm{rad} / \mathrm{s}]$.

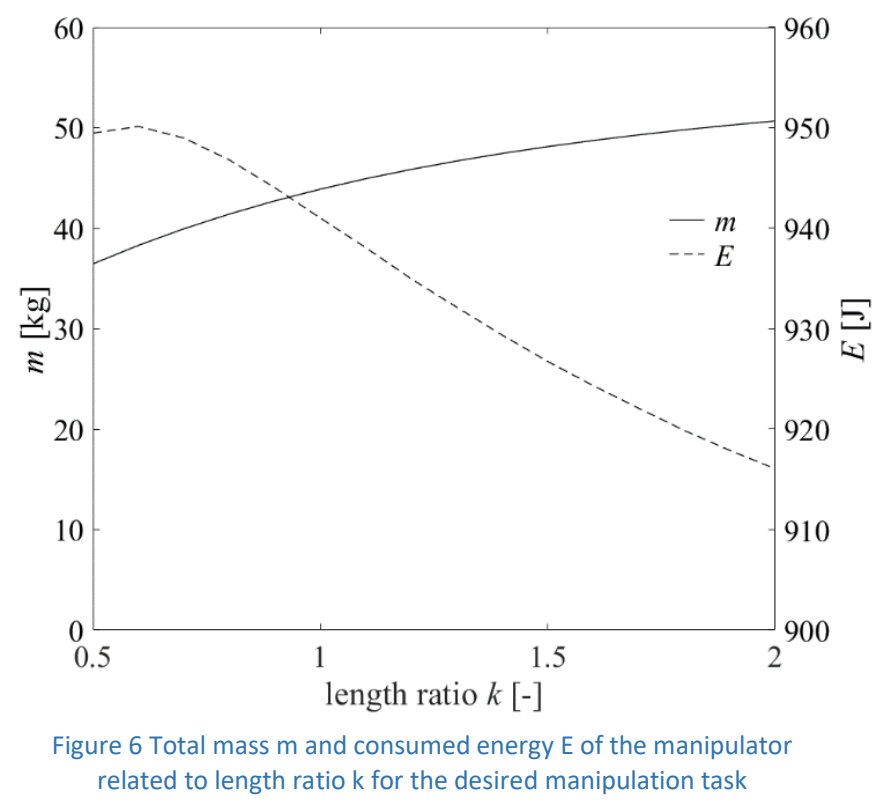

These results lead to a recommendation to select a length ratio with a longer first link and consecutively shortening other links. The relationship between energy consumption and the lengths ratio of the links is very flat, as is also shown in Figure 6, and, in contrast, it results in a better variant by consecutively extending links, but the impact of this criterion is much smaller than the impact of the criterion considering the total mass of the mechanism.

The total mass of a manipulator is mostly generated by the mass of the drive units - motors, drives and joints. In the reference mechanism the mass of armatures of joints is calculated by multiplying the motor mass by a coefficient $k_{m p}=1.5$. The weight of the drives increases significantly for higher accelerations. Figure 7 shows the course of the total weight of the manipulator as a function of the links length ratio for three different accelerations $\varepsilon\left[\mathrm{rad} / \mathrm{s}^{2}\right]$.

A reason for this behavior is the increasing influence of the reduced moment of inertia of the drives in higher angular accelerations of the links and thus of the drives, where it is necessary to choose significantly larger drives. The dependence of the required torque of the drive on a given angular acceleration is shown in Figure 8 as a multiple of the required drive torque at zero angular acceleration, i.e. only under static load. 




Figure 7 Total mass $m$ related to length ratio for 3 different angular accelerations

Today, robotic handling system are highly dynamic and achieve relatively high accelerations of a tool center point - accelerations around $20\left[\mathrm{~ms}^{-2}\right]$ are commonly used. In our simulation model, this acceleration corresponds to the angular acceleration about $\varepsilon=4[\mathrm{rad} / \mathrm{s}]$ at the highest extension of the links and thus the maximum (nominal) torque of the drive is almost double the torque needed under static load.

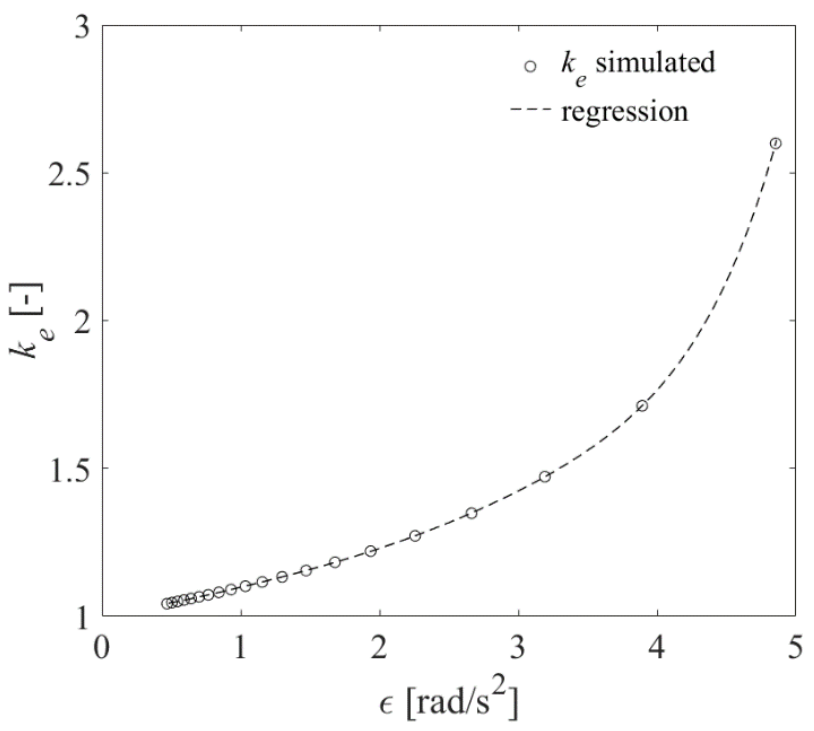

Figure 8 Coefficient of increase of needed motor torque in relation to angular acceleration

Using gear drives with higher values of moments of inertia will increase the drive size even further. Therefore, the recommended preliminary value of the coefficient of increase of the drive torque is suggested to be 1.5 to 2.0 based on known static torques. In any case it is necessary to verify a maximal value of the motor torque for the desired configuration of the manipulator, using its dynamic model that includes all reduced moments of inertia of the drives.

Finally, the search for the minimum of material and energy requirements in the manipulator synthesis has achieved partial success by finding the minimum weight of the manipulator links - in our case aluminum alloy tubes. In contrast to the overall weight trajectories depending on the links length ratio, the total mass of the all links has a minimum as is shown in Figure 9 for the acceleration of $\varepsilon=1.03[\mathrm{rad} / \mathrm{s}]$ as an example. The arm links in the simulation model consist of a tube of Al6061 material with a defined wall thickness of $2 \mathrm{~mm}$, the maximum stress was set to $100 \mathrm{MPa}$.

Similar results have also been found for other cross-sections of arm links. The minimum for weight of the arm links is not very conspicuous, only 3 to $6 \%$ of the total mass of the links, and for higher angular accelerations, this minimum of the total weight of the arm links shifts slightly to higher values of the links length ratio, i.e. towards longer links.

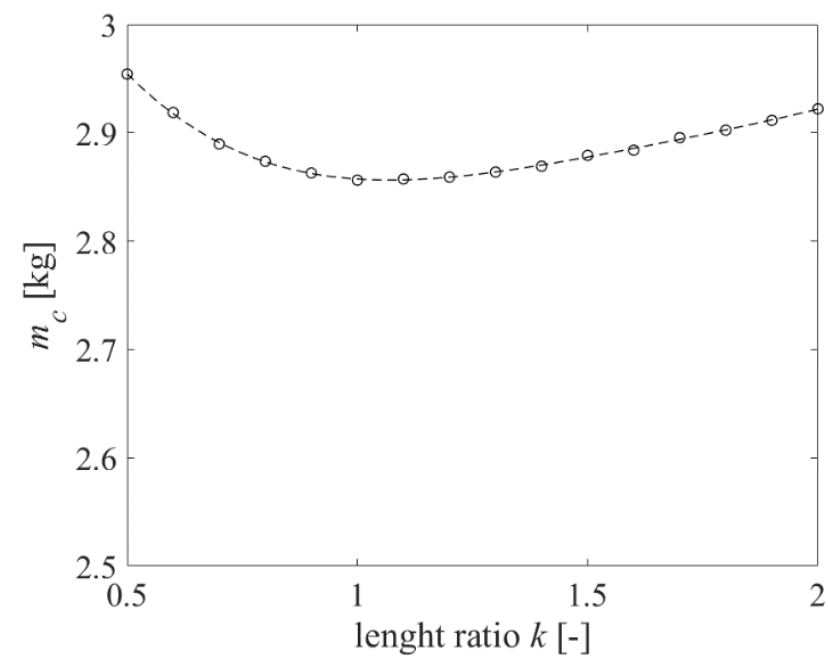

Figure 9 Total mass of the rigid-body links related to length ratio of the links

\section{CONCLUSION}

In conclusion, it can be stated that there is no unambiguous recommendation on length of links or more precisely on lengths ratio of individual links in this type of manipulator with drives in joints. The weight of such a manipulator is formed particularly by the weight of the drives, which is significantly increased by the need to overcome the reduced moment of inertia for higher angular accelerations of the links.

An insignificant minimum of the total weight of the arm links was found at approximately the same lengths of the individual links, and in consecutively lengthening links for higher accelerations. However, to optimize the total weight of the manipulator, a longer first link and consecutively shortening links are preferable. Thus, the decisive parameter remains the requirements for working space, obstacle collision avoidance in that work space and also collision avoidance between manipulator links. Only when it is possible to choose the lengths of the links is the more advantageous variant available, with consecutively shortening links. In this case it is possible to achieve at least $20 \%$ savings in the total weight of the manipulator, mainly due to significantly smaller drives of shortening links on the second, third and other axes. 


\section{ACKNOWLEDGMENTS}

This article was developed with the support of the project Research Centre of Advanced Mechatronic Systems, reg. no. CZ.02.1.01/0.0/0.0/16_019/0000867 in the frame of the Operational Program Research, Development and Education.

\section{REFERENCES}

[Björkenstam 2013] Björkenstam, S., at al. Energy efficient and collision free motion of industrial robots using optimal control. In: Proceedings of the IEEE Int. Conference on Automation Science and Engineering (CASE), 17 - 21 Aug, 2013, Madison, WI USA, 2013, pp. 510-515. doi: 10.1109/CoASE.2013.6654025S.

[Bukata 2017] L. Bukata, P. Sucha, Z. Hanzalek and P. Burget Energy Optimization of Robotic Cells. IEEE Transactions on Industrial Informatics, vol. 13, no. 1, pp. 92-102, Feb. 2017. doi: 10.1109/TII.2016.2626472

[Chen 2011] Yuan Chen and Bing Li A Piecewise AccelerationOptimal and Smooth-Jerk Trajectory Planning Method for Robot Manipulator along a Predefined Path. International Journal of Advanced Robotic Systems. Vol. 8, issue 4, pp 50, 2011. doi: $10.5772 / 45707$

[Gouasmi 2012] Gouasmi, M. et al. Kinematic Modelling and Simulation of a 2-R Robot Using SolidWorks and Verification by MATLAB/Simulink. International Journal of Advanced Robotic Systems, Volume 9, issue 6, pp 245, 2012. doi: 10.5772/50203 [Luo 2018] Luo, X., Li, S., Liu, S., \& Liu, G. (n.d.). An optimal trajectory planning method for path tracking of industrial robots. Robotica, pp. 1-19, 2018. doi:10.1017/S0263574718001145

[Perumaal 2013] Perumaal, S. and Jawahar, N. Automated Trajectory Planner of Industrial Robot for Pick-and-Place Task. International Journal of Advanced Robotic Systems, Vol. 10, issue 2, pp. 100, 2013. doi: $10.5772 / 53940$

\section{CONTACTS:}

prof. Dr. Ing. Vladimir Mostyn

VSB - Technical University of Ostrava

Faculty of Mechanical Engineering

Department of Robotics

17. listopadu 2172/15

70800 Ostrava

Czech Republic

Tel: +420597324257

vladimir.mostyn@vsb.cz

https://www.vsb.cz

https://www.fs.vsb.cz/354/cs
[Komak 2018] Komak, M., Kralik, M., Jerz, V. The generation of robot effector trajectory avoiding obstacles. MM Science Journal, 2018 (June), pp. 2367-2372.

doi: 10.17973/MMSJ.2018_06_201764

[Porawagama 2014] Porawagama, C. D., Munasinghe, S. R. Reduced jerk joint space trajectory planning method using 5-3-5 spline for robot manipulators. In: 7th International Conference on Information and Automation for Sustainability, Colombo, 2224 Dec. 2014, pp. 1-6. doi: 10.1109/ICIAFS.2014.7069580

[Pozary.cz 2011] Pozary.cz: Hasicsky robot Hardy [online]. YouTube, 4. 9. 2011,

https://www.youtube.com/watch?v=xAuOPEQiqXA

[Vergnano 2012] A. Vergnano et al. Modeling and Optimization of Energy Consumption in Cooperative Multi-Robot Systems. IEEE Transactions on Automation Science and Engineering, vol. 9, no. 2, pp. 423-428, April 2012. doi: 10.1109/TASE.2011. 2182509

[Xin 2015] Xin, G. et al. Hierarchical Kinematic Modelling and Optimal Design of a Novel Hexapod Robot with Integrated Limb Mechanism. International Journal of Advanced Robotic Systems, volume 12, issue 9 , pp 123, 2015. doi: 10.5772/59989

[Zhang 2017] Zhang Q., Yuan M. and Song R. Robot trajectory planning method based on genetic chaos optimization algorithm. In: Proceedings of the 18th Int. Conference on Advanced Robotics (ICAR), 10-12 July 2017, Hong Kong, China, pp. 602-607. doi:10.1109/ICAR.2017.8023673 